\title{
Effect of marital status on the survival of patients with gallbladder cancer treated with surgical resection: a population-based study
}

\author{
Dou-Sheng Bai ${ }^{1, *}$, Ping Chen ${ }^{1}$, Jian-Jun Qian ${ }^{1,}{ }^{,}$, Sheng-Jie Jin ${ }^{1}$, Guo-Qing Jiang ${ }^{1}$ \\ ${ }^{1}$ Department of Department of Hepatobiliary and Pancreatic Surgery, Clinical Medical College of Yangzhou University, \\ Yangzhou, China \\ *These authors are contributed equally to the work
}

Correspondence to: Guo-Qing Jiang, email: jgqing2003@hotmail.com

Keywords: gallbladder cancer, marital status, SEER, survival analysis, surgery

Received: December 28, $2016 \quad$ Accepted: February 07, $2017 \quad$ Published: February 18, 2017

Copyright: Bai et al. This is an open-access article distributed under the terms of the Creative Commons Attribution License (CC-BY), which permits unrestricted use, distribution, and reproduction in any medium, provided the original author and source are credited.

\section{ABSTRACT}

Marital status has been reported as an independent prognostic factor for survival in various cancers, but it has been rarely studied in gallbladder cancer treated by surgical resection. We retrospectively studied Surveillance, Epidemiology, and End Results (SEER) population-based data and identified 9,041 cases of gallbladder cancer with surgical treatment between 1988 and 2013. The patients were categorized according to marital status, as "married," "never married," "widowed," or "divorced/ separated." Patients in the widowed group had a higher proportion of women withingroup comparisons, a higher rate of white race, a greater proportion of older ( $\geq 60$ years) patients, more frequency of adenocarcinoma, a greater number of tumors at well/moderate pathological grading, and more prevalence at the localized SEER stage, all of which were statistically significant $(P<0.001)$. Marital status was confirmed to be an independent prognostic factor by multivariate analysis $(P<0.001)$. Married patients had higher 5-year gallbladder cancer cause-specific survival than unmarried patients $(P<\mathbf{0 . 0 0 1})$; conversely, widowed patients had the lowest gallbladder cancer cause-specific survival compared with all other patients. Conclusions marital status is an important prognostic risk factor for survival in patients with gallbladder cancer treated with surgical resection. Widowed patients have the highest risk of death compared with other groups.

\section{INTRODUCTION}

Gallbladder cancer (GBC) is the most common biliary tract neoplasm worldwide and is a rare but a fatal malignancy characterized by poor prognosis and absence of effective therapy [1]. Surgery is the only definitively curative treatment [2]. However, even after operation, the rate of locoregional recurrence is high. It has considerable wide geographic and ethnic variation with distinctive pockets of high incidence in Eastern and Central Europe, South and Central America, South Asia, and Japan [3, 4].

Recent literature has demonstrated that marital status is an independent prognostic factor for survival in many cancers [5-8]. Wang et al. reported that marital status was an important risk predictor in pancreatic cancer and that widowed patients were at the highest risk for cancer-specific mortality [7]. Li et al. found that widowed patients with colorectal cancer were at highest risk for death compared with other groups [8]. Few study explored the effect of marital status on GBC survival. Therefore, this study aimed to investigate the relationship between marital status and GBC survival. We selected data from the Surveillance, Epidemiology, and End Results (SEER) cancer registry to study the effect of marital status on GBC cause-specific survival (GCSS) in patients with GBC treated by surgical resection.

\section{RESULTS}

Baseline patient characteristics

The present study identified 9,041 eligible patients during the 25-year study period (between 1988 and 2013), including 2,453 male and 6,588 female patients. 
Of these, $2,549(28.2 \%)$ were widowed, 4,632 (51.2\%) were married, $1,093(12.1 \%)$ had never married, and $767(8.5 \%)$ were divorced/separated. Within group comparisons, the widowed group had the higher proportion of women $(90.4 \%)$, white race $(81.4 \%)$, older ( $\geq 60$ years) patients $(96.2 \%)$, adenocarcinoma $(91.1 \%)$, and tumors at well/moderate pathological grading $(50.9 \%)$ and at localized SEER stage (48.5\%), all of which were statistically significant $(P<0.001)$. Table 1 showed the baseline patient demographics and tumor characteristics.

\section{Effect of marital status on GCSS}

The married group had higher 5-year GCSS than that of the unmarried patients $(21.1 \%$ vs. $16.1 \%$, $P<0.001$ ) (Figure 1). The 5-year GCSS was $13.9 \%$ in the widowed group, which was the lowest compared with that in the married group $(21.1 \%)$, in the never married group $(20.2 \%)$, and in the divorced/separated group (18.7\%); all differences were significant according to the univariate log rank test (all $P<0.001$ ) (Figure 2A). Black race $(P<0.001)$, older age $(P<0.001)$, early year of diagnosis $(1988-1996)(P<0.001)$, adenosquamous carcinoma $(P<0.001)$, poor or undifferentiated pathology grade $(P<0.001)$, tumor size $>5 \mathrm{~cm}(P<0.001)$, TNM stage III/IV disease $(P<0.001)$, SEER distant stage $(P<0.001)$, and the widowed group $(P<0.001)$ were found as significant risk predictor for poor survival on univariate analysis (Table 2). When multivariate survival analysis was performed, all the aforementioned variables were validated as independent risk predictors associated with poor survival (Table 2), as follows: age ( $\geq 60$ years, hazard ratio [HR] 1.521, 95\% confidence interval [CI] 1.429-1.618), race (black, HR 1.055, 95\% CI 0.973-1.144; other races, HR 0.917, 95\% CI 0.855-0.994), year of diagnosis (1997-2005, HR 0.930, 95\% CI 0.875-0.988; 2006-2013, HR 0.854, 95\% CI 0.789-0.923), histotype (squamous cell carcinoma, HR 1.551, 95\% CI 1.241-1.937, adenosquamous carcinoma, HR 1.211, 95\% CI 1.064-1.378, other, HR 1.109, 95\% CI 1.004-1.225), pathology grade (poor or undifferentiated tumor, HR 1.499, 95\% CI 1.424-1.578, unknown pathology grade, HR 0.998, 95\% CI 0.919-1.085), TNM stage (stage III/IV, HR 1.137, 95\% CI 0.972-1.329; unknown stage, HR 1.259, 95\% CI 1.091-1.452), tumor size (3-5 cm tumor, HR 1.123, 95\% CI 1.008-1.250; >5 cm tumor, HR $1.184,95 \%$ CI 1.044-1.344; unstated tumor size, HR 1.415, 95\% CI 1.301-1.539), SEER stage (regional stage, HR 1.956, 95\% CI 1.842-2.077; distant stage, HR 3.370, 95\% CI 3.160-3.594; unstaged, HR 1.719, 95\% CI 1.382-2.139), marital status (married, HR 0.774, 95\% CI 0.732-0.817; never married, HR $0.914,95 \%$ CI 0.842-0.994; divorced/ separated, HR 0.891, 95\% CI 0.813-0.977).

\section{Subgroup analysis of pathological grading}

We further analyzed the effects of marital status on survival in tumors of different pathological gradings. There were no significant differences in the subgroup of pathological gradings among the different marital status groups (Table 1), and we observed two interesting findings. On the one hand, pathological grading was an independent factor for poor survival, both in the univariate and multivariate analysis $(P<0.001)$. On the other hand, widowed patients had the lowest survival rate in comparisons at all pathological grading: For well/ moderate pathological grading tumors, 5-year GCSS of widowed patients had $9.8 \%, 10 \%$, and $6.4 \%$ reductions compared with that of married patients, never married patients, and divorced/separated patients respectively (all $P<0.01$ ). For poor/anaplastic pathological grading tumors, widowed patients had a $3.9 \%$ reduction in 5-year GCSS compared with married patients $(P<0.001)$, a $2.3 \%$ reduction in 5-year GCSS compared with never married patients $(P=0.064)$, and a $4.2 \%$ reduction in 5-year GCSS compared with divorced/separated patients $(P=0.005)$. (Table 3, and Figure 2B-2C).

\section{Subgroup analysis of SEER stage}

We also analyzed the effects of marital status on survival at each SEER stage. Again, we had two interesting findings. On the one hand, marital status was an independent risk factor for poor survival in patients with each SEER stage disease, both in the univariate and multivariate analysis $(P<0.001)$. On the other hand, widowed patients again had the lowest survival rate in comparisons at all SEER stages: For localized stage tumors, widowed patients had a $15.9 \%$ reduction in 5 -year GCSS compared with married patients ( $23.5 \%$ vs. $39.4 \%)(P<0.001)$, a $10.1 \%$ reduction in 5 -year GCSS compared with never married patients ( $23.5 \%$ vs. $33.6 \%)$ $(P<0.001)$, and a $12.6 \%$ reduction in 5 -year GCSS compared with divorced/separated patients $(23.5 \%$ vs. $36.1 \%)(P<0.001)$. For regional stage tumors, widowed patients had a $4.2 \%$ reduction in 5-year GCSS compared with married patients $(6.7 \%$ vs. $10.9 \%)(P<0.001)$, a $5.9 \%$ reduction in 5-year GCSS compared with never married patients $(6.7 \%$ vs. $12.6 \%)(P=0.010)$, and an $3.1 \%$ reduction in 5-year GCSS compared with divorced/ separated patients $(6.7 \%$ vs. $9.8 \%)(P=0.015)$ (Table 4 , and Figure 2D-2F).

\section{DISCUSSION}

Some studies have suggested married patients have longer overall survival and lower mortality than those who have never married, separated, widowed, or divorced for many important causes of death respectively [13-15]. By 
Table 1: Baseline demographic and tumor characteristics of gallbladder cancer patients in the SEER database

\begin{tabular}{|c|c|c|c|c|c|c|}
\hline Characteristic & Total & Widowed & Married & Never married & $\begin{array}{l}\text { Divorced/ } \\
\text { separated }\end{array}$ & $P$ \\
\hline & $(n=9041)$ & $\begin{array}{c}(n=2549) \\
\quad N(\%)\end{array}$ & $\begin{array}{c}(n=4632) \\
N(\%)\end{array}$ & $\begin{array}{c}(n=1093) \\
N(\%)\end{array}$ & $\begin{array}{c}(n=767) \\
N(\%)\end{array}$ & \\
\hline Sex & & & & & & $<0.001$ \\
\hline Male & $2453(27.1)$ & $245(9.6)$ & $1760(38.0)$ & $283(25.9)$ & $165(21.5)$ & \\
\hline Female & $6588(72.9)$ & $2304(90.4)$ & $2872(62.0)$ & $810(74.1)$ & $602(78.5)$ & \\
\hline Age & & & & & & $<0.001$ \\
\hline$<60$ & $1917(21.2)$ & $96(3.8)$ & $1171(25.3)$ & $406(37.1)$ & $244(31.8)$ & \\
\hline$\geq 60$ & $7124(78.8)$ & $2453(96.2)$ & $3461(74.7)$ & $687(62.9)$ & $523(68.2)$ & \\
\hline Race & & & & & & $<0.001$ \\
\hline White & $7154(79.1)$ & $2074(81.4)$ & $3664(79.1)$ & $812(74.3)$ & $604(78.7)$ & \\
\hline Black & $844(9.3)$ & $203(8.0)$ & $324(7.0)$ & $197(18.0)$ & $120(15.6)$ & \\
\hline Other* & $1043(11.5)$ & $272(10.7)$ & $644(13.9)$ & $84(7.7)$ & $43(5.6)$ & \\
\hline Year of diagnosis ${ }^{\dagger}$ & & & & & & $<0.001$ \\
\hline 1988-1996 & $1832(20.3)$ & $637(25.0)$ & $928(20.0)$ & $153(14.0)$ & $114(14.9)$ & \\
\hline $1997-2005$ & $3459(38.3)$ & $1002(39.3)$ & $1765(38.1)$ & $393(36.0)$ & $299(39.0)$ & \\
\hline $2006-2013$ & $3750(41.5)$ & $910(35.7)$ & $1939(41.9)$ & $547(50.0)$ & $354(46.2)$ & \\
\hline Histotype & & & & & & 0.027 \\
\hline Adenocarcinoma & $8135(90.0)$ & $2323(91.1)$ & $4149(89.6)$ & $984(90.0)$ & $679(88.5)$ & \\
\hline $\begin{array}{l}\text { Squamous cell } \\
\text { carcinoma }\end{array}$ & $93(1.0)$ & $32(1.3)$ & $43(0.9)$ & $10(0.9)$ & $8(1.0)$ & \\
\hline $\begin{array}{l}\text { Adenosquamous } \\
\text { carcinoma }\end{array}$ & $276(3.1)$ & $73(2.9)$ & $151(3.3)$ & $35(3.2)$ & $17(2.2)$ & \\
\hline Other" & $537(5.9)$ & $121(4.7)$ & $289(6.2)$ & $64(5.9)$ & $63(8.2)$ & \\
\hline $\begin{array}{l}\text { Pathological } \\
\text { grading }\end{array}$ & & & & & & 0.354 \\
\hline Well/moderate & $4632(51.2)$ & $1297(50.9)$ & $2385(51.5)$ & $583(53.3)$ & $367(47.8)$ & \\
\hline Poor/anaplastic & $3498(38.7)$ & $992(38.9)$ & $1774(38.3)$ & $409(37.4)$ & $323(42.1)$ & \\
\hline Unknown & $911(10.1)$ & $260(10.2)$ & $473(10.2)$ & $101(9.2)$ & $77(10.0)$ & \\
\hline TNM stage & & & & & & $<0.001$ \\
\hline $\mathrm{I} / \mathrm{II}$ & $768(8.5)$ & $211(8.3)$ & $369(8.0)$ & $126(11.5)$ & $62(8.1)$ & \\
\hline III/IV & $1072(11.9)$ & $232(9.1)$ & $579(12.5)$ & $156(14.3)$ & $105(13.7)$ & \\
\hline Unknown & 7201 (79.6) & $2106(82.6)$ & $3684(79.5)$ & $811(74.2)$ & $600(78.2)$ & \\
\hline \multicolumn{7}{|l|}{ Tumor size } \\
\hline$<3 \mathrm{~cm}$ & $1356(15.0)$ & $331(13.0)$ & $708(15.3)$ & $192(17.6)$ & $125(16.3)$ & $<0.001$ \\
\hline $3-5 \mathrm{~cm}$ & $891(9.9)$ & $232(9.1)$ & $450(9.7)$ & $108(9.9)$ & $101(13.2)$ & \\
\hline$>5 \mathrm{~cm}$ & $524(5.8)$ & $125(4.9)$ & $268(5.8)$ & $81(7.4)$ & $50(6.5)$ & \\
\hline Not stated & $6270(69.4)$ & $1861(73.0)$ & $3206(69.2)$ & $712(65.1)$ & $491(64.0)$ & \\
\hline SEER stage & & & & & & $<0.001$ \\
\hline Localized & $3994(44.2)$ & $1236(48.5)$ & $1951(42.1)$ & $502(45.9)$ & $305(39.8)$ & \\
\hline Regional & $2564(28.4)$ & $715(28.1)$ & $1328(28.7)$ & $294(26.9)$ & $227(29.6)$ & \\
\hline Distant & $2386(26.4)$ & $576(22.6)$ & $1297(28.0)$ & $284(26.0)$ & $229(29.9)$ & \\
\hline Unstaged & $97(1.1)$ & $22(0.9)$ & $56(1.2)$ & $13(1.2)$ & $6(0.8)$ & \\
\hline
\end{tabular}


Abbreviations: SEER, Surveillance, Epidemiology, and End Results.

*Other includes American Indian/Alaska native, Asian/Pacific Islander, and unknown.

the early and middle Year of diagnosis were all lasted nine years, the latter was lasted eight years

\#Other cancers includes signet ring, small cell, giant and spindle cell, non-small cell carcinoma, carcinoma not otherwise specified, or undifferentiated carcinoma.

using the SEER database to determine the relationship between marital status and survival, the present study showed that married patients had significantly better GCSS than their unmarried counterparts. Widowed patients had the lowest GCSS compared with all other patients. Moreover, in multivariable analyses, the risk for widowed patients lasted even after adjusting for age, race, year of diagnosis, histologic type, pathological grading, tumor size, TNM stage, and SEER stage.

One hypothesis for the bad prognosis in unmarried individuals has delayed diagnosis with advanced tumor stage; however, in this study group, Table 1 showed the percentages of patients with well/moderate and poor/ anaplastic pathological gradings were comparable among the four subgroups. Moreover, widowed patients had the highest rate of well/moderate pathological grading. Widowed patients had worse 5-year GCSS (17.9\%) compared with married (27.7\%), never married (27.9\%), and divorced/separated $(24.3 \%)$ patients (all $P<0.01$ ). Similarly, among the patients with poor/anaplastic pathological grading, the widowed group had worse 5-year LCSS (7.1\%) compared with married $(11.0 \%)$ and divorced/separated $(11.3 \%)$ patients (all $P<0.01$ ). Notably, at poor/anaplastic pathological grading, there was no significant difference in GCSS between the widowed group and never married ( $7.1 \%$ vs. $9.4 \%, P=0.064)$ - this is a result of smaller sample size.

Psychosocial factors may provide a reasonable explanation for the relationship between marital status and survival. Although psychosocial factors are regarded as an independent reason, considered separately from tumor biological characteristics and extent of treatment, these may play several important roles associated with cancer progress. Unmarried and especially widowed patients may suffer from a lack of emotional support and social attention (otherwise provided by a spouse), which contributes to more distress, depression, and anxiety than that experienced by their married counterparts [16]. Also, a cancer diagnosis can lead to distress [17]. In widowed patients, increased mortality rates may be due to the inability to relieve stress and the loss of social attention [18].

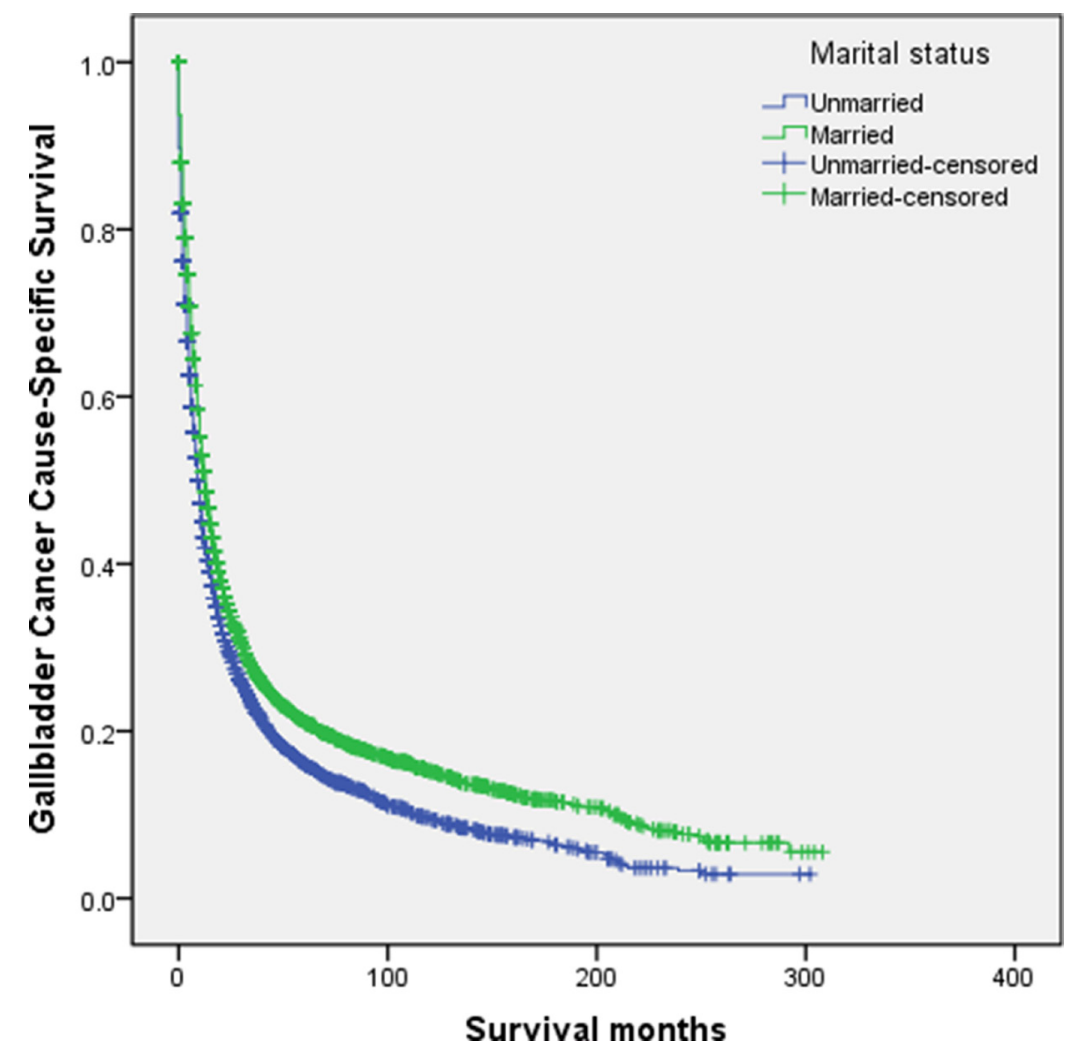

Figure 1: Survival curves in gallbladder cancer patients treated with surgical resection between the unmarried patients and the married patients. $\chi^{2}=74.829, P<0.001$. 
Table 2: Univariate and multivariate survival analysis for evaluating the influence of marital status on gallbladder cancer cause-specific survival in SEER database

\begin{tabular}{|c|c|c|c|c|c|c|}
\hline \multirow[t]{2}{*}{ Variable } & \multirow{2}{*}{$\begin{array}{l}\text { Total } \\
9041\end{array}$} & \multirow[t]{2}{*}{ 5-year CCS } & \multicolumn{2}{|c|}{ Univariate analysis } & \multicolumn{2}{|c|}{ Multivariate analysis } \\
\hline & & & $\begin{array}{c}\text { Log rank } \\
\chi 2 \text { test }\end{array}$ & $\boldsymbol{P}$ & HR(95\%CI) & $\boldsymbol{P}$ \\
\hline Sex & & & 1.770 & 0.183 & & NI \\
\hline Male & 2453 & $17.6 \%$ & & & & \\
\hline Female & 6588 & $19.1 \%$ & & & & \\
\hline Age & & & 148.198 & $<0.001$ & & $<0.001$ \\
\hline$<60$ & 1917 & $26.5 \%$ & & & Reference & \\
\hline$\geq 60$ & 7124 & $16.7 \%$ & & & $1.521(1.429-1.618)$ & \\
\hline Race & & & 19.846 & $<0.001$ & & 0.032 \\
\hline White & 7154 & $18.2 \%$ & & & Reference & \\
\hline Black & 844 & $16.9 \%$ & & & $1.055(0.973-1.144)$ & 0.191 \\
\hline Other* & 1043 & $23.8 \%$ & & & $0.917(0.855-0.994)$ & 0.034 \\
\hline Year of diagnosis ${ }^{\dagger}$ & & & 107.566 & $<0.001$ & & $<0.001$ \\
\hline 1988-1996 & 1832 & $15.5 \%$ & & & Reference & \\
\hline $1997-2005$ & 3459 & $17.0 \%$ & & & $0.930(0.875-0.988)$ & 0.019 \\
\hline 2006-2013 & 3750 & $22.4 \%$ & & & $0.854(0.789-0.923)$ & $<0.001$ \\
\hline Histotype & & & 85.488 & $<0.001$ & & $<0.001$ \\
\hline Adenocarcinoma & 8135 & $19.3 \%$ & & & Reference & \\
\hline Squamous cell carcinoma & 93 & $11.9 \%$ & & & $1.551(1.241-1.937)$ & $<0.001$ \\
\hline $\begin{array}{l}\text { Adenosquamous } \\
\text { carcinoma }\end{array}$ & 276 & $8.1 \%$ & & & $1.211(1.064-1.378)$ & 0.004 \\
\hline Other $^{\#}$ & 537 & $16.0 \%$ & & & $1.109(1.004-1.225)$ & 0.042 \\
\hline Pathological grading & & & 649.023 & $<0.001$ & & $<0.001$ \\
\hline Well/moderate & 4632 & $24.6 \%$ & & & Reference & \\
\hline Poor/anaplastic & 3498 & $9.7 \%$ & & & $1.499(1.424-1.578)$ & $<0.001$ \\
\hline Unknown & 911 & $23.4 \%$ & & & $0.998(0.919-1.085)$ & 0.970 \\
\hline TNM stage & & & 251.771 & $<0.001$ & & 0.002 \\
\hline $\mathrm{I} / \mathrm{II}$ & 768 & $52.1 \%$ & & & Reference & \\
\hline III/IV & 1072 & $15.3 \%{ }^{\dagger \dagger}$ & & & $1.137(0.972-1.329)$ & 0.109 \\
\hline Unknown & 7201 & $23.7 \%$ & & & $1.259(1.091-1.452)$ & 0.002 \\
\hline Tumor size & & & 226.873 & $<0.001$ & & $<0.001$ \\
\hline$<3 \mathrm{~cm}$ & 1356 & $32.7 \%$ & & & Reference & \\
\hline $3-5 \mathrm{~cm}$ & 891 & $21.7 \%$ & & & $1.123(1.008-1.250)$ & 0.034 \\
\hline$>5 \mathrm{~cm}$ & 524 & $16.5 \%$ & & & $1.184(1.044-1.344)$ & 0.009 \\
\hline Not stated & 6270 & $16.0 \%$ & & & $1.415(1.301-1.539)$ & $<0.001$ \\
\hline SEER stage & & & 1911.283 & $<0.001$ & & $<0.001$ \\
\hline Localized & 3994 & $33.4 \%$ & & & Reference & \\
\hline Regional & 2564 & $9.7 \%$ & & & $1.956(1.842-2.077)$ & $<0.001$ \\
\hline Distant & 2386 & $3.7 \%$ & & & $3.370(3.160-3.594)$ & $<0.001$ \\
\hline Unstaged & 97 & $12.2 \%$ & & & $1.719(1.382-2.139)$ & $<0.001$ \\
\hline Marital status & & & 105.116 & $<0.001$ & & $<0.001$ \\
\hline Widowed & 2549 & $13.9 \%$ & & & Reference & \\
\hline
\end{tabular}




\begin{tabular}{|l|l|l|l|l|l|l|}
\hline Married & 4632 & $21.1 \%$ & & & $0.774(0.732-0.817)$ & $<0.001$ \\
\hline Never married & 1093 & $20.2 \%$ & & & $0.914(0.842-0.994)$ & 0.035 \\
\hline Divorced/separated & 767 & $18.7 \%$ & & & $0.891(0.813-0.977)$ & 0.014 \\
\hline
\end{tabular}

Abbreviations: SEER, Surveillance, Epidemiology, and End Results; CCS, cause-specific survival.

*Other includes American Indian/Alaska native, Asian/Pacific Islander, and unknown.

tThe early and middle Year of diagnosis were all lasted nine years, the latter was lasted eight years

${ }^{*}$ Other cancers includes signet ring, small cell, giant and spindle cell, non-small cell carcinoma, carcinoma not otherwise specified, or undifferentiated carcinoma.

ॠ3-year CCS. Because TNM stage record according to the AJCC Cancer Staging Manual (7th edition) in the SEER database began from 2009, and ended at 2013, its 5-year CCS did not exist.

NI: not included in the multivariate survival analysis.

Furthermore, the level of adherence to the treatment plan may be different due to marital status. Compared with unmarried patients, married patients were inclined to be more likely to comply with treatment [19]; conversely, unrecognized clinical depression may lead to poor adherence to medical treatment and, further, that married patients showed a lower risk of major depression [20].

There is some evidence that the level of physiological stress and depression may affect cancer outcomes via different mechanisms. Increased psychological stress and decreased psychosocial support may contribute to weakened immune function and, in this way, may lead to tumor progression and mortality [21-23]. Reportedly, two meta-analyses showed that depression increased cancer mortality by $19 \%$ and $39 \%$, respectively [24, 25]. Furthermore, perceived lack of social support has been proven to destroy the activity of natural killer cells [26]. Also, chronic stress may contribute to downregulated cortisol receptors in white blood cells [27]. This downregulation also degrades the cellular response to anti-inflammatory signals and accelerates cytokinemediated inflammatory processes [28], which, in colorectal cancer, has been regarded as a poor prognostic factor $[29,30]$. Additionally, a previous study reported that some
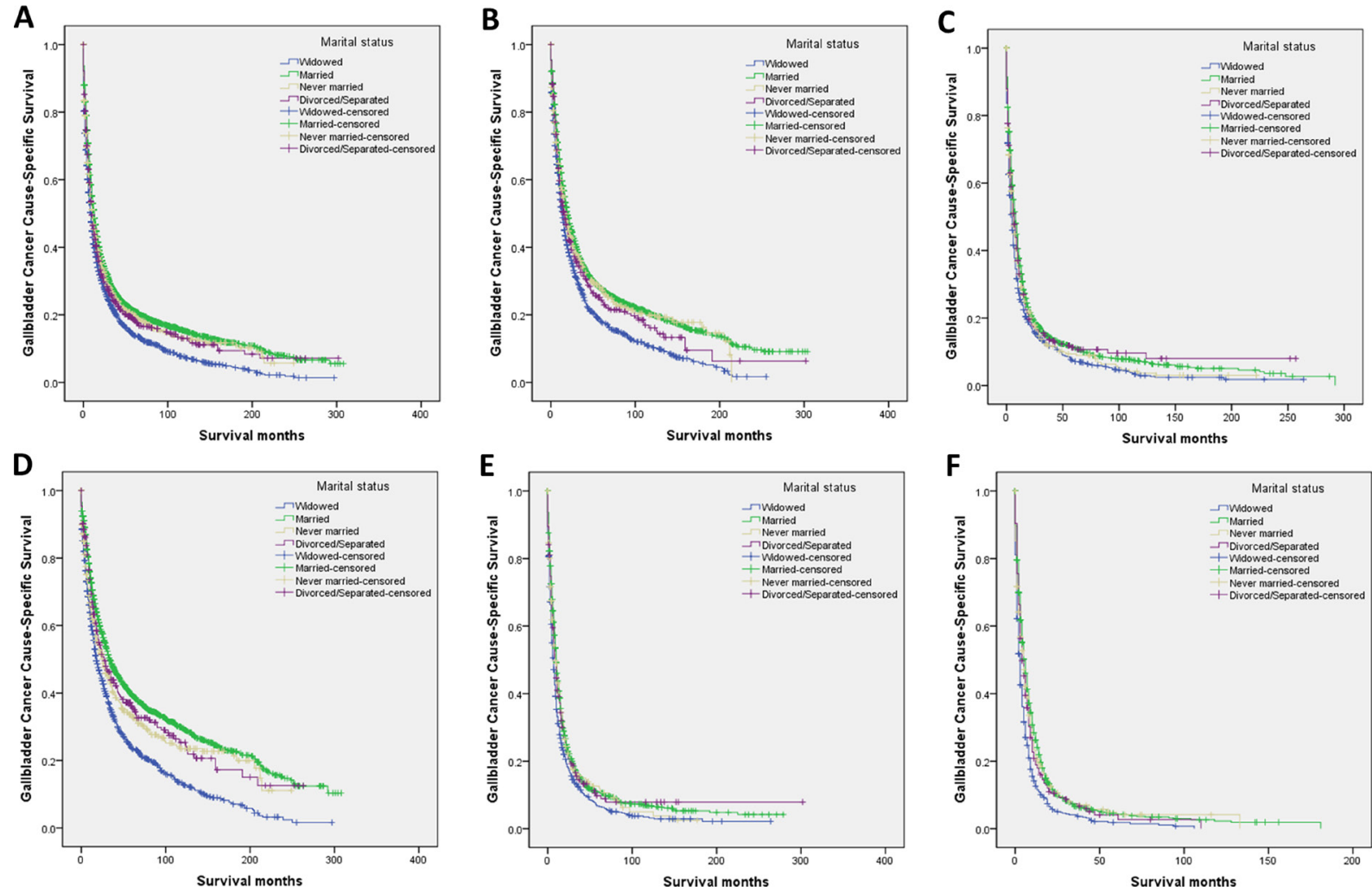

Figure 2: Survival curves in gallbladder cancer patients treated with surgical resection according to marital status. (A) Overall: $\chi^{2}=113.429, P<0.001$; (B) Well/moderate: $\chi^{2}=69.264, P<0.001$; (C) Poor/anaplastic: $\chi^{2}=37.754, P<0.001$; (D) Localized: $\chi^{2}=124.013, P<0.001 ;(\mathbf{E})$ Regional: $\chi 2=29.216, P<0.001 ;(\mathbf{F})$ Distant: $\chi^{2}=68.705, P<0.001$. 
Table 3: Univariate and multivariate analysis of marital status on gallbladder cancer cause-specific survival based on different pathological grading

\begin{tabular}{|c|c|c|c|c|c|c|}
\hline \multirow[t]{2}{*}{ Variable } & \multirow[t]{2}{*}{ Total } & \multirow{2}{*}{$\begin{array}{c}\text { 5-year } \\
\text { CCS }\end{array}$} & \multicolumn{2}{|c|}{ Univariate analysis } & \multicolumn{2}{|c|}{ Multivariate analysis } \\
\hline & & & Log rank $\chi^{2}$ test & $\boldsymbol{P}$ & HR(95\%CI) & $\boldsymbol{P}$ \\
\hline Pathological gradin & & & & & & \\
\hline Well/moderate & 4632 & & & & & \\
\hline Marital status & & & 69.264 & $<0.001$ & & $<0.001$ \\
\hline Widowed & 1297 & $17.9 \%$ & Reference & & Reference & \\
\hline Married & 2385 & $27.7 \%$ & 68.612 & $<0.001$ & $0.727(0.674-0.786)$ & $<0.001$ \\
\hline Never married & 583 & $27.9 \%$ & 18.276 & $<0.001$ & $0.780(0.695-0.877)$ & $<0.001$ \\
\hline Divorced/separated & 367 & $24.3 \%$ & 7.422 & 0.006 & $0.832(0.728-0.951)$ & 0.007 \\
\hline Poor/anaplastic & 3498 & & & & & \\
\hline Marital status & & & 37.754 & $<0.001$ & & $<0.001$ \\
\hline Widowed & 992 & $7.1 \%$ & Reference & & Reference & \\
\hline Married & 1774 & $11.0 \%$ & 37.118 & $<0.001$ & $0.784(0.722-0.851)$ & $<0.001$ \\
\hline Never married & 409 & $9.4 \%$ & 3.437 & 0.064 & $0.891(0.788-1.008)$ & 0.067 \\
\hline Divorced/separated & 323 & $11.3 \%$ & 7.772 & 0.005 & $0.827(0.723-0.946)$ & 0.006 \\
\hline
\end{tabular}

Abbreviations: CCS, cause-specific survival.

other neuroendocrine mediators and cytokines present in depression, and stress had been associated with cancer metastasis [23]. Finally, depression and poor quality of life may lead to an increased level of vascular endothelial growth, which may promote endothelial cell migration, proliferation, and proteolytic activity [31].

The present study investigated SEER data to evaluate the relationship between marital status and the postoperative prognosis of GBC; however, the study had some potential limitations. First, the SEER database only provided marital status at diagnosis. Marital status may have changed for some patients during the therapeutic process, and these changes may have affected the outcomes. Second, some data of marital status may have been inexhaustive - for example; some married patients may have separated, while other never married patients may have been cohabitating. Third, the quality of a marriage can also affect the survival of GBC patients. Marital distress has also been associated with long-term immune consequences and has contributed to an elevated risk of various health problems [32]. Finally, the SEER GBC database lacks quality data on adjuvant therapy, comorbidities, and recurrence.

To our best knowledge, this is the first report studying the effect of marital status on the survival of GBC patients treated with surgical resection. Despite these potential limitations, our study confirmed that unmarried patients are at greater risk for cancer-specific mortality. Furthermore, we showed that widowed patients were always at the highest risk for death via cancer. Psychosocial factors may be the primary reasons leading to poor survival in unmarried patients. Therefore, to improve postoperative survival, physicians should take into consideration social supports during their care of unmarried patients with GBC and especially of widowed patients. Further clinical trials should be performed to confirm our hypothesis.

\section{Statistical analysis}

We analyzed sex, age, race, primary tumor site, histologic type, pathology grade, tumor size, TNM stage, SEER stage, survival months, vital status, and marital status at the time of diagnosis. The TNM stage according to the criteria described at the American Joint Committee on Cancer (AJCC) Cancer Staging Manual (7th edition) was established. We categorized patients as "never married," "married," "widowed," or "separated/divorced." "Unmarried" included "never married," "widowed," and "separated/divorced." The individuals who were separated and who were divorced were grouped together in the group in our study.

The primary focus of this study was GCSS, which was obtained from the date of diagnosis of gallbladder cancer and the date of gallbladder cancer cause-specific death. Deaths attributed to GBC were treated as events, and deaths from other causes were treated as censored observations.

The baseline patient demographics and tumor characteristics were analyzed using the chi-square test. Death rate of the GBC was evaluated between groups using the Kaplan-Meier method. Risk factors for survival outcome were assessed using multivariable Cox regression models. All statistical analyses were performed using 
Table 4: Univariate and multivariate analysis of marital status on gallbladder cancer cause-specific survival based on different SEER stage

\begin{tabular}{|c|c|c|c|c|c|c|}
\hline \multirow[t]{2}{*}{ Variable } & \multirow[t]{2}{*}{ Total } & \multirow{2}{*}{$\begin{array}{c}\text { 5-year } \\
\text { CCS }\end{array}$} & \multicolumn{2}{|c|}{ Univariate analysis } & \multicolumn{2}{|c|}{ Multivariate analysis } \\
\hline & & & Log rank $\chi^{2}$ test & $P$ & $\mathrm{HR}(95 \% \mathrm{CI})$ & $P$ \\
\hline SEER stage & & & & & & \\
\hline Localized & 3994 & & & & & \\
\hline Marital status & & & 124.013 & $<0.001$ & & $<0.001$ \\
\hline Widowed & 1236 & $23.5 \%$ & Reference & & Reference & \\
\hline Married & 1951 & $39.4 \%$ & 124.908 & $<0.001$ & $0.625(0.575-0.681)$ & $<0.001$ \\
\hline Never married & 502 & $33.6 \%$ & 16.809 & $<0.001$ & $0.771(0.679-0.876)$ & $<0.001$ \\
\hline Divorced/separated & 305 & $36.1 \%$ & 19.570 & $<0.001$ & $0.712(0.611-0.830)$ & $<0.001$ \\
\hline Regional & 2564 & & & & & \\
\hline Marital status & & & 29.216 & $<0.001$ & & $<0.001$ \\
\hline Widowed & 715 & $6.7 \%$ & Reference & & Reference & \\
\hline Married & 1328 & $10.9 \%$ & 29.036 & $<0.001$ & $0.775(0.704-0.853)$ & $<0.001$ \\
\hline Never married & 294 & $12.6 \%$ & 6.633 & 0.010 & $0.822(0.709-0.953)$ & 0.009 \\
\hline Divorced/separated & 227 & $9.8 \%$ & 5.954 & 0.015 & $0.817(0.696-0.959)$ & 0.013 \\
\hline Distant & 2386 & & & & & \\
\hline Marital status & & & 68.705 & $<0.001$ & & $<0.001$ \\
\hline Widowed & 576 & $1.9 \%$ & Reference & & Reference & \\
\hline Married & 1297 & $4.4 \%$ & 68.102 & $<0.001$ & $0.669(0.604-0.741)$ & $<0.001$ \\
\hline Never married & 284 & $4.2 \%$ & 19.727 & $<0.001$ & $0.716(0.616-0.831)$ & $<0.001$ \\
\hline Divorced/separated & 229 & $4.1 \%$ & 13.733 & $<0.001$ & $0.749(0.639-0.879)$ & $<0.001$ \\
\hline
\end{tabular}

Abbreviations: SEER, Surveillance, Epidemiology, and End Results; CCS, cause-specific survival.

the statistical software package SPSS 22.0 software (IBM Corp, Armonk, NJ, USA). A $P$ value $<0.050$ was considered statistically significant.

\section{MATERIALS AND METHODS}

\section{Baseline patient characteristics}

The SEER program of the National Cancer Institute is an authoritative source of information on cancer incidence and survival in the United States. The SEER program registries routinely collect patient clinical data including demographics, the tumor morphology and stage at diagnosis, first course of treatment, the follow-up for survival, and so on. SEER currently collects and publishes cancer incidence and survival data from 18 populationbased cancer registries that represent approximately $30 \%$ of the population in the United States.

SEER data contain no identifiers and have been widely used for studies of the relationship between marital status and survival outcome in patients with cancer [5,6,9-12]. We used SEER*Stat 8.1.5 software to identify patients with a histopathologic diagnosis of GBC between 1988 and 2013. SEER registry patients eligible for this cohort included those with the following histologic type
ICD-O-3: adenocarcinoma (8140, 8141, 8143, 8147), papillary adenocarcinoma $(8260,8261,8262,8263)$, mucinous adenocarcinoma $(8480,8481)$, adenocarcinoma with metaplasia $(8571,8572,8573,8574,8575,8576)$, papillary carcinoma $(8050,8051,8052)$, duct carcinoma $(8500,8501,8503,8504,8507,8508)$, squamous cell carcinoma (8070, 8071, 8072, 8073, 8074, 8075, 8076, $8078)$, adenosquamous carcinoma $(8560,8562)$, or other cancers, including signet ring (8490), small cell (8041, 8043), giant and spindle cell (8030-8035), non-small cell carcinoma (8046), carcinoma not otherwise specified $(8010,8011,8012,8013,8014,8015)$, or undifferentiated carcinoma $(8020,8021,8022)$. Patients with any other histologic type were excluded from analysis.

We excluded patients who were less than 18 years at diagnosis; did not undergo surgical resection for $\mathrm{GBC}$; had multiple primary cancers, of which the GBC was not the first; and who had an unknown cause of death or unknown survival length.

According to the SEER staging system, tumors that remained in situ or confined to the organ of origin were considered to be localized; tumors that invaded locally or metastasized to regional lymph nodes were regarded as regional, while those that traveled to distant organs were considered to be distant. 


\section{ACKNOWLEDGMENTS AND FUNDING}

This work was supported by six kinds of talents of jiangsu province (No. WSW-087).

\section{CONFLICTS OF INTEREST}

The authors made no disclosures.

\section{REFERENCES}

1. Miller G, Jarnagin WR. Gallbladder carcinoma. Eur J Surg Oncol. 2008; 34:306-12.

2. Reddy SK, Clary BM. Surgical management of gallbladder cancer. Surg Oncol Clin N Am. 2009; 18:307-324, ix.

3. Lazcano-Ponce EC, Miquel JF, Muñoz N, Herrero R, Ferrecio C, Wistuba II, Alonso de Ruiz P, Aristi Urista G, Nervi F. Epidemiology and Molecular Pathology of Gallbladder Cancer. CA Cancer J Clin. 2001; 51:349-364.

4. Kapoor VK. Gallbladder Cancer: A Global Perspective. J Surg Oncol. 2006; 93:607-609.

5. Denberg TD, Beaty BL, Kim FJ, Steiner JF. Marriage and ethnicity predict treatment in localized prostate carcinoma. Cancer. 2005; 103:1819-1825.

6. Nelles JL, Joseph SA, Konety BR. The impact of marriage on bladder cancer mortality. Urologic oncology. 2009; 27:263-267.

7. Wang XD, Qian JJ, Bai DS, Li ZN, Jiang GQ, Yao J. Marital status independently predicts pancreatic cancer survival in patients treated with surgical resection: an analysis of the SEER database. Oncotarget. 2016; 7:24880-24887. doi: 10.18632/oncotarget.8467.

8. Li Q, Gan L, Liang L, Li X, Cai S. The influence of marital status on stage at diagnosis and survival of patients with colorectal cancer. Oncotarget. 2015; 6:7339-7347. doi: 10.18632/oncotarget.3129.

9. Aizer AA, Chen MH, McCarthy EP, Mendu ML, Koo S, Wilhite TJ, Graham PL, Choueiri TK, Hoffman KE, Martin NE, Hu JC, Nguyen PL. Marital status and survival in patients with cancer. J Clin Oncol. 2013; 31:3869-3876.

10. Wang L, Wilson SE, Stewart DB, Hollenbeak CS. Marital status and colon cancer outcomes in US Surveillance, Epidemiology and End Results registries: does marriage affect cancer survival by gender and stage?. Cancer epidemiology. 2011; 35:417-422.

11. Rippentrop JM, Joslyn SA, Konety BR. Squamous cell carcinoma of the penis: evaluation of data from the surveillance, epidemiology, and end results program. Cancer. 2004; 101:1357-1363.

12. Kravdal H, Syse A. Changes over time in the effect of maritalstatus on cancer survival. BMC public health. 2011; 11:804.

13. Kaplan RM, Kronick RG. Marital status and longevity in the United States population. Journal of epidemiology and community health. 2006; 60:760-765.
14. Hu YR, Goldman N. Mortality differentials by marital status: an international comparison. Demography. 1990; 27:233-250.

15. Ikeda $A$, Iso $H$, Toyoshima $H$, Fujino $Y$, Mizoue $T$, Yoshimura T, Inaba Y, Tamakoshi A. JACC Study Group. Marital status and mortality among Japanese men and women: the Japan Collaborative Cohort Study. BMC public health. 2007; 7:73.

16. Goldzweig G, Andritsch E, Hubert A, Brenner B, Walach N, Perry S, Baider L. Psychological distress among male patients and male spouses: what do oncologists need to know?. Ann Oncol. 2010; 21:877-883.

17. Kaiser NC, Hartoonian N, Owen JE. Toward a cancerspecific model of psychological distress: population data from the 2003-2005 National Health Interview Surveys. J Cancer Surviv. 2010; 4:291-302.

18. Martikainen P, Valkonen T. Mortality after the death of a spouse: rates and causes of death in a large Finnish cohort. American journal of public health. 1996; 86:1087-1093.

19. Iwashyna TJ, Christakis NA. Marriage, widowhood, and health-care use. Soc Sci Med. 2003; 57:2137-2147.

20. DiMatteo MR, Lepper HS, Croghan TW. Depression is a risk factor for noncompliance with medical treatment: metaanalysis of the effects of anxiety and depression on patient adherence. Arch Intern Med. 2000; 160:2101-2107.

21. Garssen B, Goodkin K. On the role of immunological factors as mediators between psychosocial factors and cancer progression. Psychiatry research. 1999; 85:51-61.

22. Sklar LS, Anisman H. Stress and coping factors influence tumor growth. Science. 1979; 205:513-515.

23. Moreno-Smith M, Lutgendorf SK, Sood AK. Impact of stress on cancer metastasis. Future oncology. 2010; 6:1863-1881.

24. Satin JR, Linden W, Phillips MJ. Depression as a predictor of disease progression and mortality in cancer patients: a meta-analysis. Cancer. 2009; 115:5349-5361.

25. Pinquart $M$, Duberstein PR. Depression and cancer mortality: a meta-analysis. Psychological medicine. 2010; 40:1797-1810.

26. Levy SM, Herberman RB, Whiteside T, Sanzo K, Lee J, Kirkwood J. Perceived social support and tumor estrogen/ progesterone receptor status as predictors of natural killer cell activity in breast cancer patients. Psychosomatic medicine. 1990; 52:73-85.

27. McEwen BS. Physiology and neurobiology of stress and adaptation: central role of the brain. Physiological reviews. 2007; 87:873-904.

28. Miller GE, Cohen S, Ritchey AK. Chronic psychological stress and the regulation of pro-inflammatory cytokines: a glucocorticoid-resistance model. Health psychology. 2002; 21:531-541.

29. Formica V, Luccchetti J, Cunningham D, Smyth EC, Ferroni P, Nardecchia A, Tesauro M, Cereda V, Guadagni F, Roselli M. Systemic inflammation, as measured by 
the neutrophil/lymphocyte ratio, may have differential prognostic impact before and during treatment with fluorouracil, irinotecan and bevacizumab in metastatic colorectal cancer patients. Med Oncol. 2014; 31:166.

30. Hamilton TD, Leugner D, Kopciuk K, Dixon E, Sutherland FR, Bathe OF. Identification of prognostic inflammatory factors in colorectal liver metastases. BMC Cancer. 2014; 14:542.
31. Ferrara N, Davis-Smyth T. The biology of vascular endothelial growth factor. Endocrine reviews. 1997; 18:4-25.

32. Jaremka LM, Glaser R, Malarkey WB, Kiecolt-Glaser JK. Marital distress prospectively predicts poorer cellular immune function. Psychoneuroendocrinology. 2013; 38:2713-2719. 